\title{
Re: Maternal lipids are associated with newborn adiposity independent of GDM status, obesity and insulin resistance: a prospective observational cohort study. (First comment on BJOG-19-0616.R2)
}

\author{
Ellis Muggleton ${ }^{1}$ and Tuelin Muggleton ${ }^{1}$ \\ ${ }^{1}$ Klinikum rechts der Isar der Technischen Universität München
}

May 5, 2020

Letter to the Editor, BJOG Exchange

Re: Maternal lipids are associated with newborn adiposity independent of GDM status, obesity and insulin resistance: a prospective observational cohort study

[Authors' title] High carbohydrate Intake Influences Foetal Development

Whilst welcoming research into the metabolic influences on fetal development and birth, we do not agree with the conclusions of Samsuddin et $\mathrm{al}^{1}$ that maternal lipaemia and GWG at a low threshold adversely impact neonatal adiposity". This is, in our opinion, an incorrect interpretation of the physiological processes involved.

Although the study did demonstrate an association of triglyceride levels with large-for-gestational age, this is an association and not a causative relationship. Serum triglycerides are largely dependent on diet. This phenomenon, known as carbohydrate-induced hypertriglyceridaemia (HPTG) has been well described ${ }^{2}$. Given the high carbohydrate content of the malaysian diet, the carbohydrate intake in these patients is likely both the cause of the elevated triglyceride levels and the subsequent weight gain. High-carbohydrate diets also lead to increased synthesis of fatty acids ${ }^{3}$. The insulin sensitivity need not necessarily be reduced in this group but the total exposure to insulin (in effect the area under the curve) will be increased leading to, amongst other effects, excess growth.

Insulin sensitvity was reduced in the gestational diabetes group and in the obese group with normal glucose tolerance in comparison to the non-obese group with normal glucose tolerance. This is to be expected; obesity and high carbohydrate intake will, however, eventually lead to increased IR.

The authors focus on the role of maternal lipaemia on subsequent generations. However, insulin has been demonstrated to significantly influence placental development. Total exposure to Insulin is increased in patients consuming a high carbohydrate diet. Fetal insulin has been shown to bind to fetal endothelium and alter the expression of several genes involved in metabolism ${ }^{4}$. These changes are more likely to alter the cardiovascular risk of the foetuses in adult age.

It is our interpretation of the data that high carbohydrate nutrition is the modifiable risk-factor that needs addressing. Reduction in carbohydrate intake would lead to less significant weight gain, less insulin resistance and less exposure to insulin. This would allow an improvement in placental function and less endothelial damage in the foetus.

Ellis Muggleton and Tülin Muggleton 
Department of Anesthesiology and Intensive Care Medicine

Klinikum rechts der Isar

Technical University of Munich

REFERENCES

Samsuddin S, Arumugam PA et al. Maternal lipids are associated with newborn adiposity independent of GDM status, obesity and insulin resistance: a prospective observational cohort study. BJOG. DOI 10.1111/1471-0528.16031

Parks E.J. Effect of Dietary Carbohydrate on Triglyceride Metabolism in Humans. The Journal of Nutrition 131(10): 2772S-2774S, 2001.

Hudgins LC. Effect of high-carbohydrate feeding on troglyceride and saturated fatty acid synthesis. Proc Soc Exp Biol Med 2000 Dec; 225 (3): 178-83.

Ruiz-Palacios M, Ruiz-Alcaraz AJ et al. Role of Insulin in Placental Transport in Gestational Diabetes Mellitus. Ann Nutr Metab 2017; 70: 16-25. 